\title{
Expression of an in vitro biologically active equine LH/CG without C-terminal peptide (CTP) and/or ß26-110 disulphide bridge
}

\author{
C Galet, M Chopineau, N Martinat, Y Combarnous and \\ F Guillou
}

Laboratoire de Physiologie de la Reproduction des Mammifères Domestiques, URA INRA-CNRS 129137380 Nouzilly, France

(Requests for offprints should be addressed to C Galet; Email: galet@tours.inra.fr)

\begin{abstract}
The C-terminal region of the $\beta$ subunit of the human chorionic gonadotrophin (hCG) is implied in heterodimer stability ( $\beta 26-110$ disulphide bridge), in vitro LH bioactivity (region $\beta 102-110$ ) and in in vivo LH bioactivity $(\beta$ CTP). Like the hCG $\beta$, the equine eLH and eCG $\beta$ subunits, also possess a C-terminal extension (CTP). But, in contrast to hCG, eLH and eCG bind to both LH and FSH receptors in species other than the horse. This allows investigation of the roles of the $\beta$ subunit $\mathrm{C}$-terminal region of a eLH/CG recombinant molecule on both $\mathrm{LH}$ and FSH activities. To do so, the CTP was deleted and/or the $\beta 26-110$ disulphide bond was mutated and the resulting mutated $\beta$ subunits were transiently co-expressed with common $\alpha$ subunit in COS7 cells. These regions were also deleted in a $\beta \alpha \mathrm{eLH} / \mathrm{CG}$ single chain also expressed in COS7 cells. The hormones produced were characterized
\end{abstract}

by different ELISAs and in vitro LH and FSH bioassays. Mutation of the 26-110 disulphide bond and deletion of the $\beta C T P$ led to a decrease in eLH/CG heterodimer production. Double mutation promoted an additive effect on production of the heterodimer and of the corresponding tethered eLH/CG. The elimination of the $\beta 26-110$ disulphide bond in the $\beta \alpha$ single chain had no effect on its production. However, neither the 26-110 disulphide bond nor the CTP mutations affected dimer stability and bioactivities of the secreted heterodimers and/or single chain molecules. Therefore, in contrast to hCG, the 26-110 S-S bond of the recombinant eLH/CG $\beta$ subunit does not seem to be essential for eLH/CG dimer stability upon secretion and expressing LH and FSH bioactivities. Journal of Endocrinology (2000) 167, 117-124

\section{Introduction}

The glycoprotein hormone family consists of luteinizing hormone ( $\mathrm{LH})$, follicle-stimulating hormone $(\mathrm{FSH})$ and thyroid-stimulating hormone (TSH), which are secreted by the pituitary gland in all mammalian species, and chorionic gonadotrophin (CG), which is secreted by the placenta only in primates and equids. The gonadotrophins LH, FSH and CG are involved in the control of gonadal function. All glycoprotein hormones consist of two noncovalently associated $\alpha$ and $\beta$ subunits. Within a species, the amino acid sequence of the $\alpha$ subunit is identical for all the glycoprotein hormones and is encoded by a single gene. Only the $\alpha \beta$ dimer is active and the biological specificity is conferred by the $\beta$ subunits (Pierce \& Parsons 1981, Combarnous 1992).

Human CG (hCG) is the only gonadotrophin whose crystallographic structure has been resolved (Lapthorn et al. 1994), and all other glycoprotein hormones are thought to have the same global conformation based on the presence of conserved cysteines. Many studies have focused on the role of the hCG $\beta$ subunit in intracellular folding, stability of the $\alpha \beta$ dimer and biological activity. The CG $\beta$ subunit is distinguished among other $\beta$ subunits by the presence of a 30 amino acid extension called C-terminal peptide (CTP). The CTP of the hCG $\beta$ subunit has been shown to be involved in the half-life of the hormone (Matzuk et al. 1990). It is not implicated in in vitro biological activity but is required for the correct intracellular folding of the dimer (Matzuk et al. 1989, Muyan et al. 1996, Muyan \& Boime 1998).

Like hCG, eCG is secreted by the placenta and presents a CTP on its $\beta$ subunit. Interestingly, eLH, secreted by the pituitary, has the same amino-acid sequence as eCG since its subunits are encoded by the same common $\alpha$ gene and the same $\beta$ gene (Sherman et al. 1992). The recombinant hormone will thus be referred to as eLH/CG. Moreover, eLH/CG exhibits the peculiarity of binding to both LH and FSH receptors in species other than horses, including the donkey (Stewart et al. 1977). Previous biochemical studies on hypophyseal eLH (Bousfield et al. 1989) have shown that the chemical deletion of the CTP did not affect LH bioactivity of the hormone. By contrast, a more recent study (Min et al. 1996) has shown that elimination of the 


\section{Construction of truncated $\beta$ subunit cDNAs}

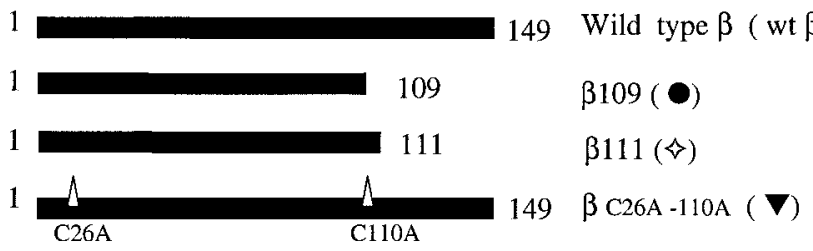

\section{Construction of $\beta \alpha$ single chain cDNAs}
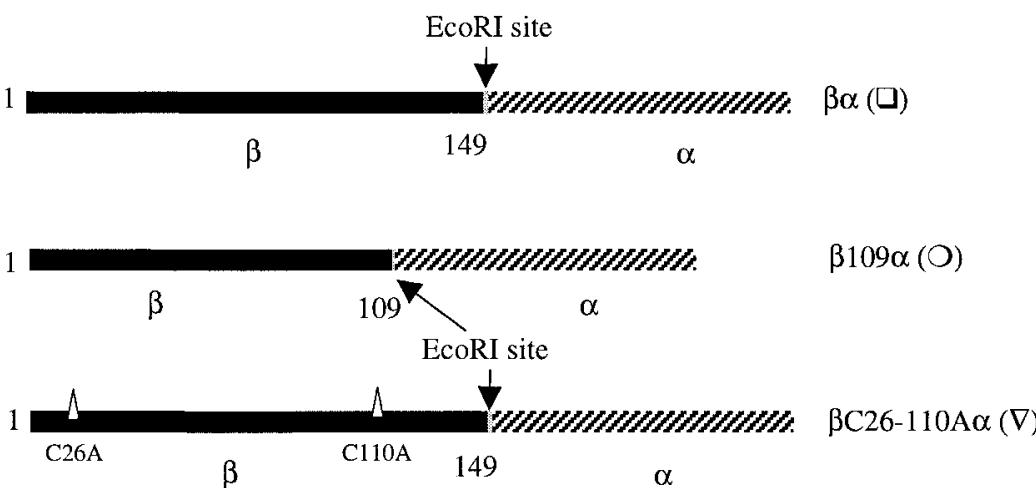

Figure 1 Schematic representation of wild-type, mutant subunits and eLH/CG single chains. A solid bar represents primary structure of $\beta$ subunit, a hatched bar represents primary structure of $\alpha$ subunit, and $\triangle$ represents mutation of cysteines 26 and 110 in alanines by site-directed mutagenesis. Symbols in parentheses are those used in Fig. 2 for each heterodimer or single chain.

$\mathrm{CTP}$ in recombinant $\mathrm{eLH} / \mathrm{CG}$ induced a tenfold increase in FSH bioactivity. So, the CTP could be crucial for correct folding of the $\beta \mathrm{eLH} / \mathrm{CG} \mathrm{C}$-terminal region (90-110 sequence) just upstream of CTP which is thought to contain biological determinants as hCG $\beta$ (Moyle et al. 1994, Han et al. 1996).

All $\beta$ subunits possess 12 cysteines at conserved positions, forming six intrachain disulphide bonds. According to the literature (Huth et al. 1993), the 26-110 disulphide bond in hCG is formed after $\alpha \beta$ dimer formation and plays an essential role in its stability and in the presentation of the $\beta 90-110$ sequence involved in the hCG biological activity (Moyle et al. 1994). The $\beta 26-110$ disulphide bridge fastens the so-called 'seat belt' formed by the 90-110 sequence of the $\beta$ subunit, which wraps around the $\alpha$ subunit. However, this $\mathrm{S}-\mathrm{S}$ bond in the hLH $\beta$ subunit which has no CTP would be formed before the interaction of $\alpha$ and $\beta$ subunits (Muyan et al. 1998), indicating that the folding stabilized by the $\beta 26-110$ disulphide bridge fastening could be different in LH and hCG and could be important for the production, stability and bioactivity of gonadotrophins.

In order to investigate the roles of CTP and the $\beta 26-110$ disulphide bridge, we studied the effect of deletion of CTP and/or the 26-110 disulphide bond in the eLH/CG $\beta$ subunit on the production, stability and bioactivities of recombinant $\alpha \beta$ dimer and $\beta \alpha$ single chain produced in COS7 cells cultures. We show that deleted recombinant $\mathrm{eLH} / \mathrm{CG}$ retains its full $\mathrm{LH}$ and $\mathrm{FSH}$ bioactivities compared with its wild-type counterpart. In contrast to hCG, the stability of the eLH/CG heterodimer in the production media appears independent of the 26-110 disulphide bond and of CTP.

\section{Materials and Methods}

Construction of mutant eLH/CG $\beta$ subunit $c D N A$ s and eLH/CG single chains

The cDNA encoding the horse LH/CG $\beta$ subunit (Chopineau et al. 1995) inserted in the expression vector CDM8 was used as a template to construct the truncated $\beta$ subunit (Fig. 1).

Two truncated $\beta$ subunits were obtained by PCR using the primers $\mathrm{A}$ and $\mathrm{B}$ or $\mathrm{C}$ (Table 1), each containing a $\mathrm{XbaI}$ restriction site for cloning.

In the first truncated $\beta$ subunit ( $\beta 109$ ), the cysteine 110 was replaced by a stop codon (TTA instead of ACA) by 
Table 1 Oligonucleotide sequence of all the primers used for the construction of cDNA for wild type, mutants and single chains. They are given with their names and single-letter abbreviation used in text

\begin{tabular}{|c|c|c|}
\hline & Primer name & Primer sequence \\
\hline A & $5^{\prime} \beta$ subunit & 5'GCTCTAGAACCAAGGATGGAGATGCTCGAGGGACT 3' \\
\hline B & $\beta$ 109stop & $\begin{array}{l}\text { 5'CGTCTAGATTAGGCCAAGGGCTGGTCTC 3' } \\
\text { stop aa109 }\end{array}$ \\
\hline $\mathrm{C}$ & $\beta 111$ stop & 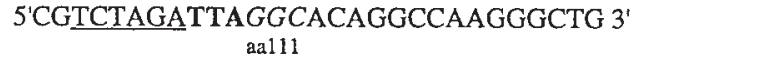 \\
\hline $\mathbf{D}$ & $\mathrm{C} 26 \mathrm{~A}$ & $\begin{array}{l}\text { 5'-GCCTGCCCCATCGCCATCACCTTCACC-3' } \\
\text { cys } \$ \text { ala }\end{array}$ \\
\hline $\mathbf{E}$ & C26A reverse & 5'-GGTGGAGGTGATGGCGATGGGGCAGGC-3' \\
\hline $\mathbf{F}$ & C110A & 5'-CCAGCCCTTGGCCGCTGCCCCCCAGGCC-3' \\
\hline G & C110A reverse & 5'-GGCCTGGGGGGCAGCGGCCAAGGGCTGG-3' \\
\hline $\mathbf{H}$ & $\beta 5^{\prime}$ end & $\begin{array}{c}\text { 5'-GCTCTAGAACCAAGGATGGAGATGCTCCAGGGACT -3' } \\
\text { Met }\end{array}$ \\
\hline I & $\beta 1493^{\prime}$ end & $\begin{array}{l}\text { 5'-CGGAATTCAGAAGTCTTTATTGGGAGGGGA-3' } \\
\text { EcoR1 Site aaß149 }\end{array}$ \\
\hline $\mathbf{J}$ & $\alpha 5^{\prime}$ end & 5'- CGGAATTCGCTCCTGATGTGCAGGATTGCC-3' \\
\hline K & $\alpha 3^{\prime}$ end & $\begin{array}{l}\text { 5'-GCTCTAGACCTTTAAATCTTGTGGTGAT-3' } \\
\text { Stop }\end{array}$ \\
\hline $\mathbf{L}$ & $\beta 109$ 3' end & $\begin{array}{l}\text { 5'-CGGAATTCGGCCAAGGGCTGGTCTCTGAAAACCCC-3' } \\
\text { aaß109 }\end{array}$ \\
\hline
\end{tabular}

PCR using the primers $\mathrm{A}$ and $\mathrm{B}$. Thus, the resulting $\beta$ subunit lacked both CTP and the 26-110 disulphide bond.

In the second ( $\beta 111)$ the stop codon was inserted just after amino acid 111 (TTA instead of CTG) by PCR using the primers $A$ and $C$. Thus, the resulting $\beta$ subunit only lacked the CTP but retained its 26-110 disulphide bond.

Mutagenesis of cysteines 26 and 110 of the horse wild-type $\beta$ LH/CG subunit, subcloned into pBluescript vector (Stratagene, Cambridge, UK) via their XbaI sites, was performed by extension of mutagenic oligonucleotides containing the mutation of cysteine 26 and/or 110 for an alanine. This was carried out using a site-directed mutagenesis kit (Quick-change site-directed mutagenesis kit, Stratagene) (Fig. 1). The sequences of the primers used to obtain the mutation in position 26 are given in Table 1 (primers D and $\mathrm{E}$ ). The primers $\mathrm{F}$ and $\mathrm{G}$ were used to obtain the mutation in 110 using the same methods.

eLH/CG single chain was constructed using an EcoRI restriction site to join the $3^{\prime}$ extremity of $\beta$ subunit cDNA to the $5^{\prime}$ extremity of $\alpha$ subunit cDNA ( $\beta \alpha$ single chain). First, the $\beta$ eLH/CG cDNA was amplified by PCR using the primers $\mathrm{H}$ and I (Table 1 ). The $\alpha$ subunit cDNA was amplified by PCR using the primers $\mathrm{J}$ and $\mathrm{K}$.

After amplification, $\beta$ and $\alpha$ eLH/CG cDNAs were fused via the EcoR1 site then the $\beta \alpha$ single-chain was subcloned into the CDM8 vector via its $\mathrm{XbaI}$ sites. The signal sequence of the $\alpha$ subunit has been deleted from the single chain and the signal sequence of the $\beta$ subunit is used to direct production of tethered eLH/CG.

In this single chain, the 110-149 region was deleted from the $\beta$ subunit by PCR using the primer $\mathrm{H}$ and the primer L. The $\beta 109 \mathrm{cDNA}$ was then fused to the $\alpha \mathrm{cDNA}$ ( $\beta 109 \alpha$ single chain) as described above. The cysteines 26 and 110 of the $\beta$ subunit were also mutated in tethered eLH/CG. This was carried out as described above for the $\beta$ subunit.

Truncated and mutant $\beta$ eLH/CG and single chain cDNAs were subcloned into the expression vector pCDM8 via their XbaI restriction sites. All constructs were amplified in MC1061/P3 bacteria (Invitrogen BV, Groningen, The Netherlands) and purified using the Qiagen maxiprep plasmid kit (Coger, Paris, France).

The sequences of all resulting constructs were checked by double-stranded DNA sequencing.

\section{Transient transfections of COS7 cells}

COS7 monkey kidney cells (ATCC-CRL 1651) were co-transfected at $65 \%$ confluence in $6 \mathrm{~cm}$ diameter Petri dishes with $2 \mu \mathrm{g}$ pCDM $8 \alpha-\mathrm{eLH}$ and $2 \mu \mathrm{g}$ pCDM $8 \beta-\mathrm{eLH}$ or with $2 \mu \mathrm{g}$ of one of the three mutated $\beta$ subunit constructs using a calcium phosphate precipitation procedure as previously described (Chopineau et al. 1997). For single-chain chimeras, COS7 cells were transfected with $4 \mu \mathrm{g}$ pCDM8 $\beta \alpha \mathrm{eLH} / \mathrm{CG}$. The cells were incubated 
with $3 \mathrm{ml}$ serum-free DMEM for $48 \mathrm{~h}$. The media were then harvested, centrifuged and the supernatants stored at $-20{ }^{\circ} \mathrm{C}$ until analysis.

The supernatants from transfected COS7 cells were quantified using the ELISA described below before being assayed in the in vitro bioassays. Supernatants were concentrated four times with Centricon YM 10 (Amicon, Millipore Corporation, Bedford, MA, USA) when the secreted amounts of recombinant hormone were too low to permit accurate measurement of the activities.

\section{Quantitation of expressed hormones}

Three different ELISAs were used to quantify the expressed hormones.

First, the quantity of recombinant wild-type, mutant and single chain horse LH/CG present in the harvested media was estimated in a dimer-specific sandwich ELISA using two monoclonal antibodies (mAbs) directed against eCG (one anti $\alpha, 89 \mathrm{~A} 2$ and one anti $\beta, 24 \mathrm{~A} 3$ ) as previously described (Chopineau et al. 1993, 1997) and eCG FL652 (5000 UI/mg), purified in our laboratory as the reference preparation. As this assay is dimer-specific, none of the free subunits cross-react, allowing only the detection of assembled subunits.

A second sandwich ELISA was used to quantify single chains and to detect the dimer and free $\alpha$ subunit in the supernatant from COS7 co-transfected with $\alpha$ and $\beta$ subunit cDNAs. Briefly, the first antibody (anti- $\alpha$ 89A2) was coated on to the microtitration plate. After washing and saturation, increasing concentrations of standard eCG or the sample were added. After $1 \mathrm{~h}$ at $37^{\circ} \mathrm{C}$, the excess of hormone was washed and a rabbit polyclonal antibody directed against eCG (Cahoreau \& Combarnous 1987), which also recognizes free subunit in our ELISA system, was added. After $1 \mathrm{~h}$ at $37^{\circ} \mathrm{C}$ and washing, the plate was incubated with a $1 / 1000^{\mathrm{e}}$ dilution of a horseradish peroxidase linked anti-rabbit antibody for $1 \mathrm{~h}$ at $37^{\circ} \mathrm{C}$. The plates were washed again and the substrate (ophenylenediamine $)$ added $(50 \mu \mathrm{g} / 100 \mu \mathrm{l} 100 \mathrm{mM}$ sodium acetate $\mathrm{pH} 5.6$ and $10 \mu \mathrm{l} \mathrm{H}_{2} \mathrm{O}_{2}$ ) and the absorbance measured at $492 \mathrm{~nm}$ after $15 \mathrm{~min}$ at room temperature. This assay detects free $\alpha$ subunit at a level of $35 \%(w / w)$, in addition to the heterodimer. $\alpha$ subunit expressed alone in COS7 cells is correctly secreted and detected in the medium by this sandwich ELISA at a range of $20 \%$ compared with the wild-type heterodimer. The difference between these two sandwich ELISAs allows the measurement of the free $\alpha$ subunit.

As all the results are based on the estimation of the production by immunoassay, a third ELISA was used to confirm the estimated quantities by the two sandwiches. This is a competitive ELISA using the anti- $\alpha$ mAb 89A2 detecting total $\alpha$, free plus combined. As previously described by Chopineau et al. (1993), this mAb presents $63 \% \mathrm{w} / \mathrm{w}$ of cross-reaction with free $\alpha$ subunit. As this
$\mathrm{mAb}$ is anti- $\alpha$, as we never mutated the $\alpha$ subunit and as this $\mathrm{mAb}$ recognizes free and combined $\alpha$ subunits, we can reasonably assume that its epitope is always intact on all the recombinant products.

\section{Estimation of LH bioactivity}

LH bioactivity of recombinant wild type and mutant LH/CG was estimated by measuring the testosterone production in a rat Leydig cell bioassay (Guillou et al. 1985). Briefly, Leydig cells from 52-day-old Wistar rats were dispersed with collagenase and incubated for $4 \mathrm{~h}$ at $34{ }^{\circ} \mathrm{C}$ in Leibowitz L15 medium, with the samples of recombinant hormones being tested at different concentrations based on ELISA estimates. After incubation, the media were harvested, centrifuged and supernatants were stored at $-20{ }^{\circ} \mathrm{C}$ until testosterone assay in a specific RIA.

\section{Estimation of FSH bioactivity}

FSH bioactivity was assessed in an in vitro progesterone stimulation assay using Y1 cell line from a mouse adrenal cortex tumour stably expressing the human FSH receptor (a kind gift from Ares Serono, Geneva, Switzerland) (Kelton et al. 1992). Approximately 150000 cells were incubated in $1 \mathrm{ml}$ growth medium (Ham's F10, 15\% horse serum (HS), 2.5\% foetal bovine serum (FBS), 1\% Gln, $80 \mu \mathrm{g} / \mathrm{ml} \mathrm{Geneticin} \mathrm{(G418)} \mathrm{and} 2.5 \mu \mathrm{g} / \mathrm{ml}$ fungizone) in 24-well plates for 2 days. The medium was changed and the cells incubated for another 2 days and then incubated with $1 \mathrm{ml}$ medium containing less serum (Ham's F10, 5\% HS, $0.8 \%$ FBS, $1 \%$ Gln, $80 \mu \mathrm{g} / \mathrm{ml} \mathrm{G} 418$ and $2.5 \mu \mathrm{g} / \mathrm{ml}$ fungizone) for $24 \mathrm{~h}$ before the final $4-\mathrm{h}$ incubation in $800 \mu \mathrm{l}$ of stimulation medium (Ham's F10, $1 \mathrm{mg} / \mathrm{ml} \mathrm{BSA}$, $1 \%$ Gln, $80 \mu \mathrm{g} / \mathrm{ml}$ G418) plus $200 \mu \mathrm{l}$ COS7 medium containing recombinant hormones. Finally, the media were harvested, boiled for $10 \mathrm{~min}$, centrifuged and the supernatants were collected in glass-heated tubes and assayed for progesterone using a specific RIA (Saumande et al. 1985).

\section{Statistical analysis}

Five expressions of each mutant were achieved. All immunoassays and bioassays were done once or twice for each expression. Statistical analyses were carried out using a paired $t$-test.

\section{Results}

Elimination of the 26-110 disulphide bond and CTP of $\beta$ subunit influence the production of the mutated hormones

Deletion of the CTP leads to a $50 \%$ diminution in the detection of mutant $\alpha \beta 111$ heterodimer which can not be ascribed to dissociation since no more free $\alpha$ subunit could 
Table 2 Production of all constructs estimated by the three different ELISA systems: (1) specific dimer $\alpha \beta$ ELISA, (2) free $\alpha$ and $\alpha \beta$ sandwich ELISA and (3) free and combined $\alpha$-specific ELISA. For each construction, three to five expressions were carried out. The quantity of each mutant is given as a percentage \pm S.E.M. of the wild-type $\alpha \mathrm{e} \beta \mathrm{e}$

Specific dimer ELISA

(\%)

\section{Constructions}

$\alpha$ e $\beta$ e

$\alpha \mathrm{e} \beta 109$

$\alpha \mathrm{e} \beta 111$

$\alpha$ e $\beta$ e $26-110 \mathrm{~A}$

Single chain $\beta \alpha$

Single chain $\beta 109 \alpha$

Single chain $\beta C 26-110 A \alpha$

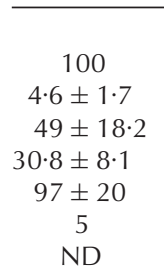

Free $\alpha+\alpha \beta$ sandwich ELISA (\%)

$\begin{aligned} & 100 \\ & 8 \cdot 6 \pm 4 \cdot 3 \\ & 55 \pm 18 \cdot 9 \\ & 29 \cdot 8 \pm 7 \cdot 4 \\ & 105 \pm 31 \cdot 4 \\ & 9 \cdot 2 \pm 2 \cdot 4 \\ & 85 \pm 28 \cdot 4\end{aligned}$

Free and combined $\alpha$ displacement ELISA

(\%)

$$
\begin{gathered}
100 \\
16 \cdot 75 \pm 9 \cdot 3 \\
77 \cdot 3 \pm 8 \cdot 1 \\
30 \pm 9 \\
\text { ND } \\
\text { ND } \\
\text { ND }
\end{gathered}
$$

ND: not determined.

be demonstrated for the mutated dimer compared with the wild type (Table 2), even if in the third ELISA, the level of $\alpha$ subunit seems overestimated. A drastic decrease in the production of the mutant $\alpha \beta 109$ in which both the CTP and the 26-110 disulphide bond were deleted was shown by the different ELISAs (Table 2). The analysis of the three ELISAs shows that there is no more free $\alpha$ subunit in the $\alpha \beta 109$ medium than in the wild-type medium. Quantitation of corresponding single-chain shows that full-length $\beta \alpha$ was produced at the same level as wildtype $\alpha \beta$ dimer, whereas the production of the $\beta 109 \alpha$ single chain was only tenfold less than the $\alpha \beta 109$ dimer (Table 2).

To determine precisely the role of the 26-110 disulphide bond on the formation of recombinant eLH/CG, cysteines 26 and 110 of the wild-type $\beta$ subunit were mutated into alanine by site-directed mutagenesis ( $\beta$ C26$110 \mathrm{~A})$. This disulphide bridge was also eliminated in the $\beta \alpha$ single chain hormone $(\beta C 26-110 A \alpha)$. The elimination of $\beta 26-110$ disulphide bond in $\beta$ ( $\alpha \beta C 26-110 \mathrm{~A}$ dimer) led to diminished detection (30\%) in the dimer-specific ELISA compared with the wild-type $\alpha \beta$ (Table 2). In contrast, the deletion of the bridge did not affect the production of the $\beta C 26-110 A \alpha$ single chain hormone (85\% compared with the $\alpha \beta$ wild type). Since there was no increase in free $\alpha$ subunit in the media, the low detection rate can again be attributed to a lower production of the $\alpha \beta C 26-110$ A dimer compared with the wild-type $\alpha \beta$.

The CTP and 'the fastening of the seat belt' by the 326-110 disulphide bond are not required for the bioactivities of recombinant $\mathrm{eLH} / \mathrm{CG}$

The LH and FSH bioactivities of eLH/CG were unaffected by the deletion of CTP as observed in Fig. 2A and B ( $\alpha \beta 111$ heterodimer). The FSH/LH ratio was $2 \cdot 46 \pm 0.64$ fold compared with the wild type (Table 3 ). However, this difference was not significant since the FSH bioactivity estimated for this mutant was not significantly different from wild-type FSH activity. As for $\alpha \beta 111$, the LH and FSH activities of the $\alpha \beta 109$ heterodimer (Fig. 2A and $B)$, and of the full-length $\beta \alpha$ and $\beta 109 \alpha$ single chain (Fig. 2C and D, Table 3) were detected and were identical to those of the wild type. Therefore, the bioactivities of eLH/CG were not modified by the elimination of both the CTP and the 26-110 disulphide bond. Concerning the role of the $26-110 \mathrm{~S}-\mathrm{S}$ bond strictly, as for previous mutants, $\mathrm{LH}$ and FSH activities of the $\alpha \beta \mathrm{C} 26-110 \mathrm{~A}$ dimer and the $\beta C 26-110 A \alpha$ single chain were similar to those of wild-type $\alpha \beta$ (Fig. 2, Table 3).

\section{Discussion}

In the present study, it was demonstrated that the absence of CTP and/or the 26-110 disulphide bond in the eLH/CG $\beta$ subunit does not impede its association to $\alpha$ subunit and does not reduce its $\mathrm{LH}$ and FSH bioactivities. Nevertheless, it severely reduces its production without affecting its quaternary structure upon secretion.

These results on eLH/CG are partially in agreement with those concerning hCG and allow comparison of these two gonadotrophins. Many studies have addressed the role of hCG CTP but, though having about the same length ( $\approx 30$ amino acids) and the same characteristics as that of eLH/CG (several O-linked sugars), it exhibits an amino acid sequence which largely differs from that of eLH/CG. As observed here for eLH/CG, deletion of hCG $\beta$ CTP has no effect on heterodimer formation and in vitro bioactivity (El Deiry et al. 1989). However, the in vivo activity of this hormone was much reduced, in accordance with the fact that the $O$-linked oligosaccharides present on the CTP extension are important for the half-life of the hormone (Matzuk et al. 1987, 1990). The fusion of a $\beta C T P$ to hFSH $\beta$ subunit had no effect on its in vitro activity but enhanced its in vivo half-life and consequently its in vivo activity. Interestingly, it also increased its 
LH bioactivity

A

eLH/CG heterodimers
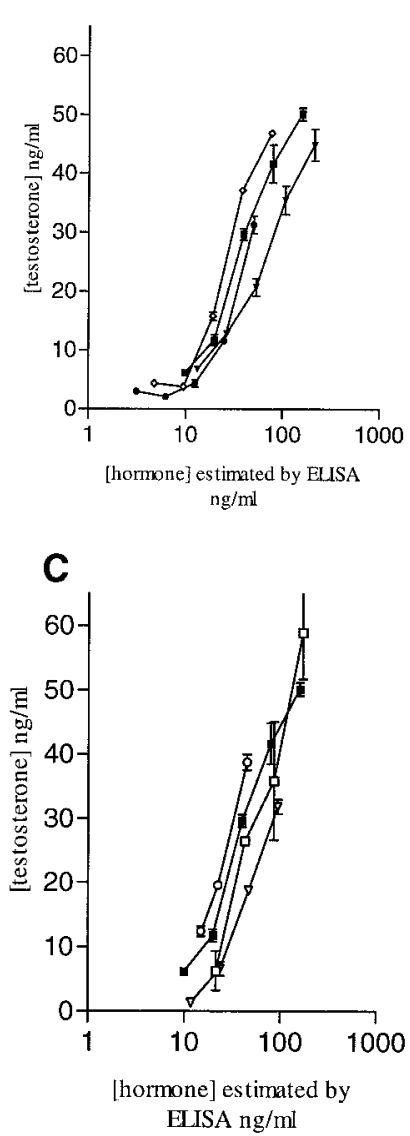

FSH bioactivity

B

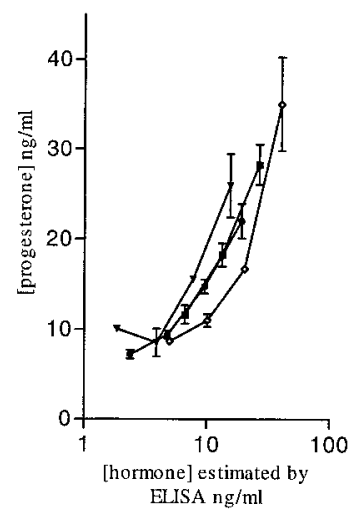

D

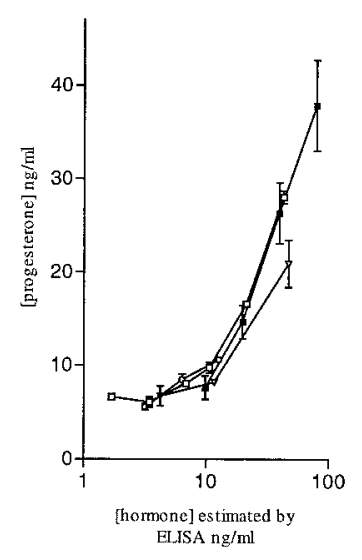

Figure $2 \mathrm{LH}$ and $\mathrm{FSH}$ bioactivities of each eLH/CG mutant and single chain. Estimation of LH bioactivity was carried out by stimulation of rat Leydig cells. For each heterodimer (A) and $\mathrm{eLH} / \mathrm{CG}$ single chain $(\mathrm{C})$, the production of testosterone was represented as a function of quantities of each one estimated by ELISA. Each point is the mean \pm S.E.M. from one representative experiment. The estimation of $\mathrm{FSH}$ bioactivity of heterodimer (B) and tethered eLH/CG (D) was carried out by stimulation of Y1 cells. After stimulation, progesterone was assayed with a specific RIA and expressed as a function of quantities of each mutant or wild-type recombinant eCG estimated by ELISA. Each point is the mean \pm S.E.M. from one representative experiment. $\alpha \mathrm{e} \beta \mathrm{e} \mathbf{\square} ; \alpha \mathrm{e} \beta 111 \diamond ; \alpha \mathrm{e} \beta 109$ $\alpha$ e $\beta$ eC26-110A $\nabla ; \beta \alpha$ single chain $\square ; \beta 109 \alpha \bigcirc ; \beta C 26-110 A \alpha \nabla$.

production rate (Fares et al. 1992). Elimination of the $\beta$ CTP in a tethered $\beta \alpha$ hCG did not affect its in vitro bioactivity but lowered its production by fivefold compared with full-length tethered $\beta \alpha h C G$ (Narayan et al. 1995).

In the present study, the elimination of the CTP had no effect on in vitro $\mathrm{LH}$ and FSH bioactivities of the eLH/CG dimer. This agrees with a study of Bousfield et al. (1989) on pituitary $\mathrm{eLH}$, in which the CTP was removed by selective acid hydrolysis (eLH des 121-149) and which exhibited the same in vitro LH activity as native eLH (its FSH activity was not determined). In a more recent study
(Min et al. 1996), the elimination of $\beta$ CTP in recombinant eLH/CG (des $115-149=\alpha \beta 114)$ was shown to increase its FSH activity by tenfold compared with wild-type hormone without changing its $\mathrm{LH}$ activity. The authors suggested a negative role for $O$-linked oligosaccharides in the FSH activity of eLH/CG. In the present study, elimination of the $\beta 112-149$ region had no effect on $\mathrm{LH}$ bioactivity of eLH/CG (Table 2, Fig. 2) but did not significantly increase its FSH bioactivity. Since the mutations do not match exactly in the two studies, one interpretation would be that residues 112-114 might be important for FSH activity. Another explanation is that the 
Table $3 \mathrm{LH}$ and FSH bioactivities of mutated recombinant eLH/CG. Estimated LH and FSH activities represent the hormone concentration $(\mathrm{ng} / \mathrm{ml})$ that yields half-maximal stimulation of testosterone production for LH bioactivity and/or progesterone for FSH bioactivity. The $\mathrm{LH}$ and FSH estimates are from four to seven experiments (mean \pm S.E.M.) each conducted in duplicate

\begin{tabular}{|c|c|c|c|}
\hline & $\begin{array}{l}\text { LH } \\
(\mathrm{ng} / \mathrm{ml})\end{array}$ & $\begin{array}{l}\text { FSH } \\
(\mathrm{ng} / \mathrm{ml})\end{array}$ & FSH/LH \\
\hline \multicolumn{4}{|l|}{ Constructions } \\
\hline$\alpha \mathrm{e} \beta \mathrm{e}$ & $20 \cdot 12 \pm 11 \cdot 3$ & $26 \pm 11 \cdot 7$ & $1 \cdot 3 \pm 0 \cdot 35$ \\
\hline$\alpha e \beta 109$ & $21 \cdot 9 \pm 14 \cdot 6$ & $23 \cdot 02 \pm 13 \cdot 3$ & $1 \cdot 05 \pm 0 \cdot 13$ \\
\hline$\alpha \mathrm{e} \beta 111$ & $15 \cdot 1 \pm 4 \cdot 6$ & $37 \cdot 11 \pm 6 \cdot 2$ & $2 \cdot 46 \pm 0 \cdot 64$ \\
\hline$\alpha \mathrm{e} \beta \mathrm{e} C 26-110 \mathrm{~A}$ & $15 \cdot 9 \pm 7 \cdot 7$ & $19 \cdot 72 \pm 6 \cdot 4$ & $1 \cdot 3 \pm 0.53$ \\
\hline Single chain $\beta \alpha$ & $25 \cdot 9 \pm 11 \cdot 7$ & $37 \cdot 3 \pm 2 \cdot 34$ & $1 \cdot 44 \pm 0 \cdot 3$ \\
\hline Single chain $\beta 109 \alpha$ & $14 \cdot 46 \pm 4 \cdot 8$ & $28 \cdot 73 \pm 15$ & $1 \cdot 92 \pm 0.54$ \\
\hline Single chain $\beta C 26-110 A \alpha$ & $31 \cdot 3 \pm 8 \cdot 65$ & $42 \cdot 05 \pm 4 \cdot 95$ & $1 \cdot 34 \pm 0 \cdot 04$ \\
\hline
\end{tabular}

two molecules differ because they were expressed in CHO cells in Min et al. (1996) vs COS7 cells in the present study, leading to different $\mathrm{N}$-saccharide chains. Finally, the difference could also originate from the fact that the FSH bioassays used were different. The CTP of eLH/CG could possibly interfere with FSH binding in the primary rat granulosa cell system used by Min et al. (1996) but not in the Y1 cell line expressing the human FSH receptor that we used.

As in the case of CTP, the absence of a 26-110 disulphide bridge had no effect on eLH/CG bioactivities. Most studies did not investigate the bioactivity of 26-110 $\mathrm{C} \rightarrow \mathrm{A}$ mutants because the amount recovered in the medium was too small. However, tethered gonadotrophins have recently been constructed to bypass the problem of assembly, and a single chain hCG $\beta \alpha$ mutated in 26-110 was shown to exhibit full $\mathrm{LH}$ activity in vitro compared with the one-chain molecule with an intact 26-110 disulphide bridge (Ben-Menahem et al. 1997). Our data confirm that this bridge is not required for LH bioactivity but is also not indispensable for the FSH bioactivity of eLH/CG. More surprisingly, this result indicates that the fastening of the 26-110 bridge is not mandatory for the assembly of $\alpha \mathrm{eLH} / \mathrm{CG}$ and $\beta \mathrm{eLH} / \mathrm{CG}$ which is required for the expression of its LH and FSH activities. By contrast, the disruption of this bond in hTSH abolished its bioactivity (Medeiros-Neto et al. 1996).

A recent paper (Jackson et al. 1999) proposed that all the quaternary structure of hCG was not required for its in vitro bioactivity. The present data would, at least in part, support this view. Nevertheless, elimination of CTP and/or the 26-110 S-S bond leads to a decreased production of the heterodimer of both mutated dimers $(\alpha \beta 109$, $\alpha \beta 111, \alpha \beta C 26-110 \mathrm{~A})$ and truncated one-chain gonadotrophin $(\beta 109 \alpha)$. This decrease could not be ascribed to a defect in heterodimer assembly since no free $\alpha$ subunit was detected in the culture medium by the different ELISA systems used (ELISAs 2 and 3, Table 2), keeping in mind that $\alpha$ subunit expressed alone in COS7 cells is well secreted and detected by these immunoassays at a range of $20 \%$ (data not shown). Moreover, the different ELISAs used an anti- $\alpha \mathrm{mAb}$ and this subunit was never mutated, so we could reasonably assume that its epitope is always intact. It is now well established that the CTP and the 26-110 disulphide bridge of the hCG $\beta$ subunit are involved in the intracellular trafficking and secretion of the hCG heterodimer (Suganuma et al. 1989, Huth et al. 1993, Muyan et al. 1996, Ruddon et al. 1996). It seems to be the same for eLH/CG, particularly for the 26-110 disulphide bridge since its deletion induced a severe decrease in the heterodimer production but no decrease in the $\beta \alpha$ single chain production (Table 1). Our hypothesis based on previous data (Feng et al. 1996, Ruddon \& Bedows 1997) is that in the case of the 26-110 mutated heterodimer, the majority of the hormone is not correctly folded for secretion, whereas the 26-110 mutated single chain takes the correct conformation required for secretion. Studies are now under way to determine how CTP and/or the 26-110 disulphide bridge influence synthesis and/or secretion of the hormone.

\section{Acknowledgements}

The authors are grateful to D Aguer (Intervet International, Boxmeer, The Netherlands) for the gift of anti-eCG monoclonal antibodies, and to A Ythier (Ares Serono, Geneva, Switzerland) for the gift of Y1 cells stably expressing the human FSH receptor, F Lecompte (INRA, Nouzilly, France) for standard eCG FL652 and M Pelloile (INRA, Nouzilly, France) for sequencing the cDNAs. C Galet is recipient of a grant from Région Centre and INRA.

\section{References}

Ben-Menahem D, Kudo M, Pixley M, Sato A, Suganuma N, Perlas E, Hsueh AJW \& Boime I 1997 The biologic action of single-chain 
choriogonadotropin is not dependent on the individual disulphide bonds of the $\beta$ subunit. Journal of Biological Chemistry 272 6827-6830.

Bousfield GR, Liu WK \& Ward DN 1989 Effect of removal of carboxy-terminal extension from equine luteinizing hormone (LH) $\beta$-subunit on LH and follicle-stimulating hormone receptor-binding activities and LH steroidogenic activity in rat testicular Leydig cells. Endocrinology 124 379-387.

Cahoreau C \& Combarnous Y 1987 Comparison of two reference preparations for horse chorionic gonadotrophin in four in vivo and in vitro assays. Journal of Reproduction and Fertility 79 281-287.

Chopineau M, Maurel MC, Combarnous Y \& Durand P 1993 Topography of equine chorionic gonadotropin epitopes relative to luteinizing hormone and follicle stimulating hormone receptor interaction sites. Molecular and Cellular Endocrinology 92 229-239.

Chopineau M, Stewart F \& Allen WR 1995 Cloning and analysis of the cDNA encoding the horse and donkey luteinizing hormone $\beta$-subunits. Gene 160 253-256.

Chopineau M, Martinat N, Marichatou H, Troipoux C, Auge-Gouillou C, Stewart F, Combarnous Y \& Guillou F 1997 Expression of horse and donkey LH in COS-7 cells: evidence for low FSH activity in donkey LH compared with horse LH. Journal of Endocrinology 152 371-377.

Combarnous Y 1992 Molecular basis of the specificity of binding of glycoprotein hormones to their receptor. Endocrine Reviews $\mathbf{1 3}$ 670-691.

El Deiry S, Kaetzel D, Kennedy J, Nilson J \& Puett D 1989 Sitedirected mutagenesis of the human chorionic gonadotropin $\beta$-subunit: bioactivity of a heterologous hormone, bovine $\alpha$-human des-(122-145)ß. Molecular Endocrinology 3 1523-1528.

Fares FA, Suganuma N, Nishimori K, LaPolt PS, Hsueh AJW \& Boime I 1992 Design of a long-acting follitropin agonist by fusing the $\mathrm{C}$-terminal sequence of the chorionic gonadotropin $\beta$ subunit to the follitropin $\beta$ subunit. PNAS 89 4304-4308.

Feng W, Bedows E, Norton SE \& Ruddon RW 1996 Novel covalent chaperone complexes associated with human chorionic gonadotropin $\beta$ subunit folding intermediates. Journal of Biological Chemistry 271 18543-18548.

Guillou F, Martinat N \& Combarnous Y 1985 Rapid in vitro desensitization of the testosterone response in rat Leydig cells by sub-active concentrations of porcine luteinizing hormone. FEBS Letter 184 6-9.

Han Y, Bernard MP \& Moyle WR 1996 hCG $\beta$ residues 94-96 alter LH activity without appearing to make Key receptor contacts. Molecular and Cellular Endocrinology 124 151-161.

Huth JR, Perini F, Lockridge O \& Ruddon WR 1993 Protein folding and assembly in vitro parallel intracellular folding and assembly. Journal of Biological Chemistry 268 16472-16482.

Jackson AM, Berger P, Pixley M, Klein C, Hsueh AJW \& Boime I 1999 The biological action of choriogonadotropin is not dependent on the complete native quaternary interactions between the subunits. Molecular Endocrinology 13 2175-2188.

Kelton CA, Cheng SVY, Nugent NP, Schweickhardt RL, Rosenthal JL, Overton SA, Wands GD, Kuzeda JB, Luchette CA \& Chappel SC 1992 The cloning of the human follicle stimulating hormone receptor and its expression in COS-7, $\mathrm{CHO}$ and $\mathrm{Y} 1$ cells. Molecular and Cellular Endocrinology 89 141-151.

Lapthorn AJ, Harris DC, Littlejohn A, Lustbader JW, Canfield RE, Machin KJ, Morgan FJ \& Isaacs NW 1994 Crystal structure of human chorionic gonadotropin. Nature 369 455-461.

Matzuk MM, Krieger M, Corless CL \& Boime I 1987 Effect of preventing $\mathrm{O}$-glycosylation on the secretion in Chinese hamster ovary cells. PNAS 184 6354-6358.
Matzuk MM, Splanger MM, Camel M, Suganuma N \& Boime I 1989 Mutagenesis and chimeric genes define determinants in the $\beta$ subunits of human chorionic gonadotropin and lutropin for secretion and assembly. Journal of Cellular Biology 109 1429-1438.

Matzuk MM, Hsueh AJW, Lapolt P, Tsafriri A, Keene JL \& Boime I 1990 The biological role of the carboxyl-terminal extension of human chorionic gonadotropin $\beta$-subunit. Endocrinology 126 376-383.

Medeiros-Neto G, Herodotou DT, Rajan S, Kommareddi S, De Lacerda L, Sandrini R, Boguszewski MCS, Hollenberg AN, Radovick S \& Wondisford FE 1996 A circulating biologically inactive thyrotropin caused by a mutation in the beta subunit gene. Journal of Clinical Investigation 97 1250-1256.

Min KS, Hattori N, Aikawa JI \& Ogawa T 1996 Site-directed mutagenesis of recombinant equine chorionic gonadotropin/luteinizing hormone: differential role of oligosaccharides in luteinizing hormone and follicle-stimulating hormone-like activities. Endocrine Journal 43 585-593.

Moyle WR, Campbell RK, Myers RV, Bernard MP, Han Y \& Wang X 1994 Co-evolution of ligand-receptor pairs. Nature 368 251-255.

Muyan M \& Boime I 1998 The carboxyl-terminal region is a determinant for the intracellular behavior of the chorionic gonadotropin $\beta$ subunit: effects on the processing of the Asn-linked oligosaccharides. Molecular Endocrinology 12 766-772.

Muyan M, Furuhashi M, Sughara T \& Boime I 1996 The carboxyterminal region of the $\beta$-subunits of luteinizing hormone and chorionic gonadotropin differentially influence secretion and assembly of the heterodimer. Molecular Endocrinology 10 1678-1687.

Muyan M, Ruddon WR, Norton SE, Boime I \& Bedows E 1998 Dissociation of early folding events from assembly of the human lutropin $\beta$-subunit. Molecular Endocrinology 12 1640-1649.

Narayan P, Wu C \& Puett D 1995 Functional expression of yoked human chorionic gonadotropin in bacculovirus-infected insect cells. Molecular Endocrinology 9 1720-1726.

Pierce JG \& Parsons TF 1981 Glycoprotein hormones: structure and function. Annual Review of Biochemistry 50 465-495.

Ruddon RW \& Bedows E 1997 Assisted protein folding. Journal of Biological Chemistry 272 3125-3128.

Ruddon RW, Sherman SA \& Bedows E 1996 Protein folding in the endoplasmic reticulum: lessons from the human chorionic gonadotropin $\beta$ subunit. Protein Science 5 1443-1452.

Saumande J, Tamboura D \& Chupin D 1985 Changes in milk and plasma concentrations of progesterone in cows after treatment to induce superovulation and their relationships with number of ovulations and of embryos collected. Theriogenology 23 719-731.

Sherman GB, Wolfe MW, Farmerie TA, Clay CM, Threadgill DS, Sharp DC \& Nilson JH 1992 A single gene encodes the $\beta$-subunits of equine luteinizing hormone and chorionic gonadotropin. Molecular Endocrinology 6 951-959.

Stewart F, Allen WR \& Moor RM 1977 Influence of fetal genotype on the follicle-stimulating hormone: luteinizing hormone ratio of pregnant mare serum gonadotrophin. Journal of Endocrinology $\mathbf{7 3}$ 419-425.

Suganuma N, Matzuk MM \& Boime I 1989 Elimination of disulphide bonds affects assembly and secretion of the human chorionic gonadotropin $\beta$ subunit. Journal of Biological Chemistry 264 19302-19307.

Received 17 March 2000

Revised manuscript received 11 May 2000

Accepted 5 June 2000 